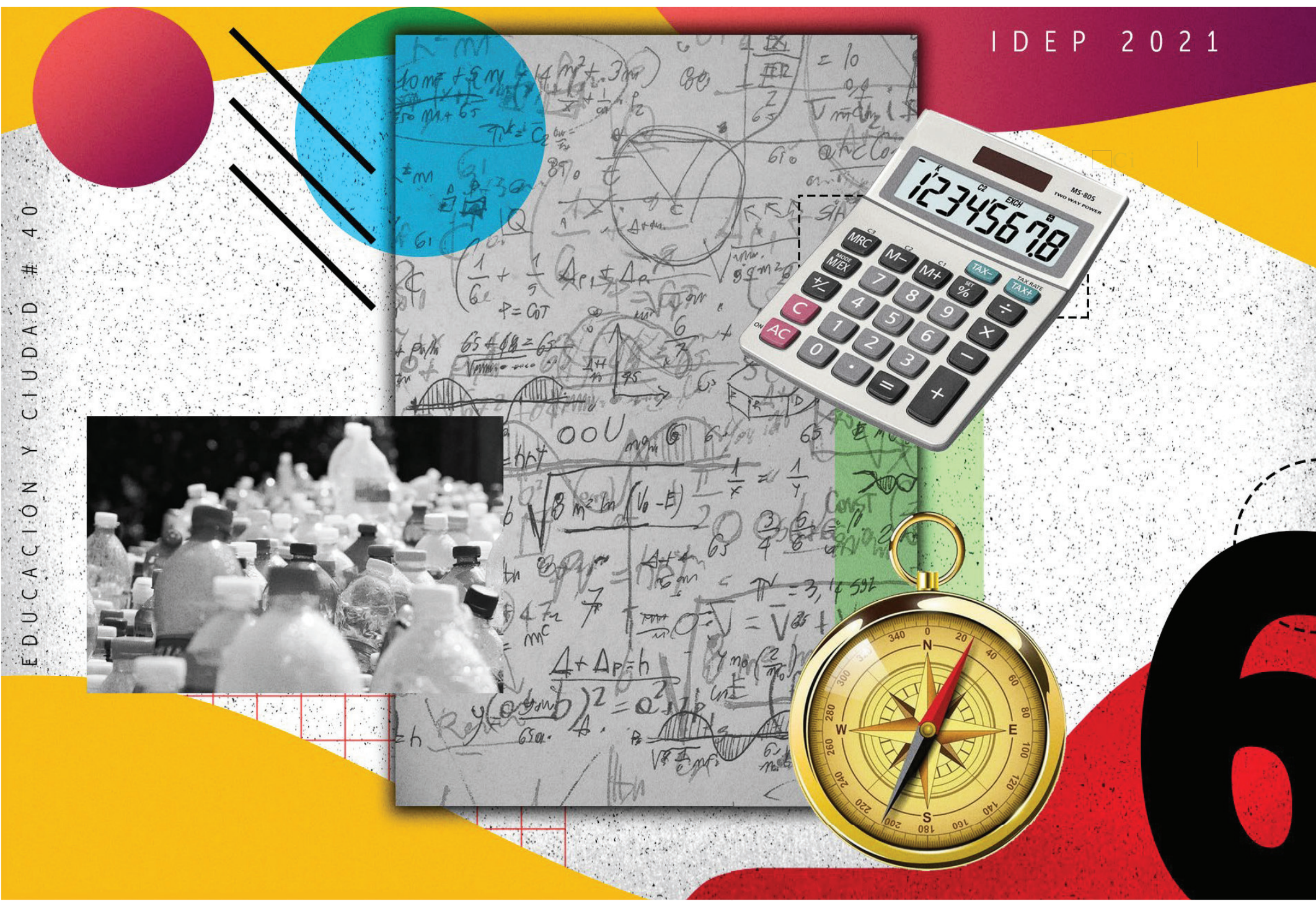

\title{
¿Y tú que desechas? Una discusión de aspectos medioambientales en la clase de matemáticas
}

WHAT ARE YOU THROWING AWAY? A DISCUSSION OF ENVIRONMENTAL ISSUES IN MATH CLASS

O QUE ESTÁS A DEITAR FORA? UMA DISCUSSÃO SOBRE

QUESTÕES AMBIENTAIS NA AULA DE MATEMÁTICA

\section{Ruben Felipe Morales Camargo}




\section{Ruben Felipe Morales Camargo ${ }^{1}$}

1. Magíster en Educación; Licenciado en Educación Básica con Énfasis en Matemáticas; ORCID: https://orcid.org/0000-0002-7668-1696; correo electrónico: rfelipe.moralesc@gmail.com

Citar artículo como:

Morales, R. (2021, Enero-Junio). ¿Y tú que desechas? Una discusión de aspectos medioambientales en la clase de matemáticas. Revista Educación y Ciudad, No. 40, pp. 99-111. //doi.org/10.36737/01230425. n40.2021.2459

DOI: https://doi.org/10.36737/01230425.n40.2021.2459

Fecha de recepción: 2 de agosto de 2020 / Fecha de aprobación: 4 de noviembre de 2020

\section{Resumen}

El presente artículo relata lo ocurrido en un escenario de aprendizaje desarrollado desde el proyecto denominado "Entropía, escuela de matemáticas", el cual, orientado desde la perspectiva de Skovsmose (1999), corresponde con lo desarrollado de manera virtual y presencial durante el primer semestre de 2020, con estudiantes de grado séptimo de una institución pública en la ciudad de Bogotá. El escenario se enmarca en la idea del manejo adecuado de residuos sólidos en los hogares, las representaciones sociales de lo ambiental, la separación de residuos y estadísticas empleadas por la Unidad Administrativa Especial de Servicios Públicos.

Palabras clave: Educación ambiental, escenarios de aprendizaje, basuras, manejo de residuos sólidos, aprendizaje de las matemáticas.

\section{Abstract}

This article relates what happened in a learning scenario developed from the project called Entropía Escuela de Matemáticas and oriented from the perspective of Skovsmose (1999); it corresponds to what was developed virtually and in person during the first semester of 2020 with seventh grade students from a public institution in the city of Bogotá. This scenario is framed within the idea of adequate solid waste management in homes, social representations of the environment, waste separation and statistics used by the Special Administrative Unit of Public Services.

Keywords: Environmental education, learning scenarios, garbage, solid waste management, learning mathematics

\section{Resumo}

Este artigo relata o que aconteceu em um cenário de aprendizagem desenvolvido a partir do projeto chamado Entropía Escuela de Matemáticas e orientado a partir da perspectiva de Skovsmose (1999); corresponde ao que foi desenvolvido virtualmente e pessoalmente durante o primeiro semestre de 2020 com estudantes da sétima série de uma instituição pública da cidade de Bogotá. Este cenário está enquadrado dentro da idéia de uma gestão adequada de resíduos sólidos em residências, representações sociais do meio ambiente, separação de resíduos e estatísticas utilizadas pela Unidade Administrativa Especial de Serviços Públicos.

Palavras-chave: Educação ambiental, cenários de aprendizagem, lixo, gestão de resíduos sólidos, aprendizagem de matemática. 


\section{A modo de introducción}

$\mathrm{U}$ no de los principales problemas de las sociedades actuales es el manejo adecuado de residuos sólidos y la disposición final de los mismos en botaderos y centros de acopio de materiales reciclables. Sin embargo, las ideas que giran alrededor de la cuestión se ven permeadas en muchas ocasiones por una deficiente educación ambiental, por patrones culturales de comportamiento, un bajo sentido de apropiación y un entendimiento de los recursos como algo ilimitado y reemplazable.

En otras ocasiones, el problema confronta posiciones respecto a la utilidad de las cosas que adquirimos y aquellas que se asumen como "necesarias"; por ejemplo, en los hogares, dada la dinámica de consumo, se cambian los electrodomésticos por unos nuevos con funciones más "avanzadas", sin utilizarlos hasta el cumplimiento de su vida útil. Esto se debe principalmente a la alta difusión comercial de algunos productos, a los altos costos de las reparaciones, en algunos casos a la obsolescencia programada, y a un gran número de otras razones que llevan a que el objeto sea reemplazado y desechado.

A diario producimos toneladas de basura que muchas veces van a parar a los botaderos y centros de reciclaje, pero también a las fuentes hídricas, alcantarillados, aceras y avenidas, generando otros problemas en el ambiente que afectan la calidad de vida de todos. Este problema no es ajeno a la escuela, donde se pueden observar grandes cantidades de basura y, en muchas ocasiones, un manejo inadecuado de los residuos, caracterizado por la no separación, desechos arrojados en lugares no destinados para ello, desaseo y malos olores derivados de la descomposición de sustancias orgánicas.
Teniendo en cuenta este escenario, junto a los estudiantes de grado séptimo del Colegio La Concepción IED (de aquí en adelante CLC), localidad de Bosa, Bogotá, se emprendió la tarea de explorar, desde la clase de matemáticas, el problema de las basuras, procurando identificar razones por las cuales es necesario clasificar, separar y dar un manejo adecuado a los desechos sólidos en casa y, al tiempo, a establecer algunas caracterizaciones posibles. Ello quiere decir que, reconociendo los alcances de la investigación, no se estableció una solución al problema, pero sí un acercamiento, viéndolo como una situación política y social que requiere de acciones concretas y que puede ser analizada desde aspectos medioambientales y matemáticos (Skovsmose, 2000).

Al respecto, es preciso señalar que al realizar una indagación preliminar con los estudiantes que participaron del proceso, respecto de lo que sucede con la basura producto de las actividades en casa, muchos de ellos manifestaron un total desconocimiento de la situación e incluso frente a la idea de separar los desechos; para muchos la basura se relaciona solo con lo que ya no sirve, independientemente de su composición, tamaño y uso.

Para otros, aquellos con familiares dedicados al reciclaje, hijos de recolectores de basura o quienes viven en la esquina de la cuadra donde los vecinos suelen dejar su bolsa cuando pasa el camión recolector, el problema de las basuras va más allá de la producción; se traduce en malos olores, presencia de roedores, mala utilización del espacio público y de los contenedores, inseguridad, problemas de salud y suciedad. 


\section{Entropía. Escuela de Matemáticas, el papel de los escenarios}

"Entropía, Escuela de Matemáticas" (en adelante EEM), es una propuesta de investigación surgida en 2015 en el CLC, para orientar el trabajo en clase de matemáticas a partir de situaciones sociales y políticas que puedan ser analizadas desde el área. El trabajo con los estudiantes implica una perspectiva socio cultural en educación matemática, donde el montaje de un escenario es una "acción intencionada del profesor para construir una situación en la que el proceso educativo pueda encarnarse para dar significado a las acciones individuales y colectivas" (Skovsmose, 1999).

En ese sentido, el trabajo desarrollado desde la EEM asume la clase como espacio de comunicación, donde tiene lugar el intercambio de ideas, presupuestos teóricos y aproximaciones conceptuales; es, además, un escenario para el encuentro con otros, así como con la posibilidad de emprender acciones en contextos socioculturales concretos. Así, se adhiere a una epistemología de las matemáticas que les entiende como prácticas sociales que enmarcan un saber matemático en tanto producto cultural, emergido en circunstancias y contextos históricos determinados.

\section{Articular las ciencias naturales y las matemáticas, trabajando desde una idea de ambiente}

En 2019, tras diferentes discusiones, el área de ciencias naturales del CLC decide orientar los desarrollos de la institución desde las asignaturas escolares de biología, química y física, a partir de la propuesta: "Enseñanza de las ciencias naturales desde proyectos de aula desarrollados por preguntas problémicas contextuales"; así mismo, estableció las temáticas a desarrollar en cada grado a partir de la generación de interrelaciones entre los contenidos a tratar y, posteriormente, la proposición de una "pregunta integradora que esté ligada al contexto de los estudiantes y que presente como características ser llamativa para su edad y presentarse como actual" (Duarte, Urazán, Quiroga, Revelo, Guevara y Gómez, 2019, p. 1006).

Basados en esta idea, y considerando investigaciones previas de los docentes del CLC, en torno a las representaciones sociales de ambiente y educación ambiental, a las Estrategias alternativas para la resignificación del concepto de ambiente (Duarte, 2014), y a la formación de líderes ambientales (García, 2017), se consideró viable asumir esta propuesta de trabajo y vincularla a lo desarrollado desde la EEM, lo cual permitió plantear la siguiente pregunta a los estudiantes: ¿Qué ocurre con las basuras que salen de nuestras casas? 


\section{Tejido teórico que permite abordar la propuesta}

La existencia de diversos enfoques en educación ambiental y en la enseñanza de las matemáticas, requiere tener claridad respecto de los presupuestos teóricos que sustentan lo desarrollado; así, en principio, es preciso describir el enfoque empleado para la educación ambiental, el cual caracteriza como un "proceso de construcción permanente de la relación con el medio ambiente a través del cual el individuo y la comunidad adquieren actitudes, compromisos y competencias" (Sauvé y Orellana, 2001, p. 52).

Así, el Proyecto Ambiental Escolar (PRAE) del CLC, expone el abordaje de la educación ambiental desde un enfoque sistémico, el cual, desde la perspectiva de Sauvé (2010), busca comprender las realidades ambientales para tomar decisiones apropiadas; al respecto, Duarte (2014) invita a abordar la educación ambiental en la escuela a partir de problemáticas contextuales que permitan realizar un trabajo reflexivo y favorezcan la construcción de la idea de ambiente en los estudiantes, de tal forma que se visualicen como parte del mismo, comprometiéndose a propiciar estrategias alternativas que garanticen mejorar su entorno.

En paralelo, vinculando lo planteado por García (2017, p. 617), es importante considerar que la educación ambiental permita a los estudiantes la posibilidad de poner en práctica un discurso actual y realista sobre las condiciones ambientales de su entorno; esto quiere decir, en términos de Sauvé (2010), que lo desarrollado con los estudiantes debe proveerles de una visión ecosistémica de las realidades ambientales, incluyendo sus componentes tecnológicos y el desarrollo de habilidades para trabajar en interdisciplinariedad (p. 8).

Sin embargo, asumir tal perspectiva sobre la educación ambiental implica también, por un lado, considerar que este tipo de educación se puede abordar en la escuela partiendo de problemáticas contextuales y, por otro, que su abordaje depende de condiciones políticas y sociales específicas, las cuales enmarcan dinámicas sociales de participación, discusión y consenso. En ese sentido, Skovsmose y Valero (2012) exponen, desde un enfoque socio político en educación matemática, la idea de colectividad, entendida desde la conciencia de que es tan necesario cooperar en la toma de decisiones y en la búsqueda de condiciones de vida favorables para todos, como emprender acciones en favor de la aplicación de las decisiones tomadas.

Así, la idea de colectividad se vincula entonces con la educación ambiental, toda vez que las acciones en pro de la reflexión y actuación de los sujetos sobre su entorno, no son individuales, sino que se alimentan de interpretaciones construidas con otro. Al respecto, Morales (2017) indica que el "Otro" es quien señala la relatividad de la propia mirada asumida sobre las cosas, ello quiere decir que al abordar una interpretación sistémica del ambiente, y reconocerse como parte de él, no solo nos vemos desde nuestro lugar de enunciación, le transformamos en el sentido de los otros que hacen parte del ambiente.

\section{Desarrollo del escenario basuras}

Algunos planteamientos de Skovsmose (1999) se refieren al escenario de investigación entendién- 
dole como aquel donde las actividades permiten al estudiante asumir el problema como propio, buscar mecanismos y alternativas de solución, situarse críticamente ante la situación y reconocer en ella su papel como agente de solución; al respecto, a continuación se describirá lo desarrollado, entendiendo el escenario basuras como espacio de investigación.

\section{Escena 1. Cómo afecta la basura a mi comunidad}

Diferentes medios de comunicación presentan el problema de las basuras como una situación que afecta a las comunidades cercanas a los botaderos dispuestos por los municipios o ciudades para alojar grandes toneladas de desechos. Últimamente, para el caso particular de Bogotá, se refieren también a los contenedores instalados por la Unidad Administrativa Especial de Servicios Públicos (UAESP), en diferentes lugares de la ciudad, para evitar acumulaciones de residuos en la vía pública. Dicha referencia se da para señalar, no solo el uso inadecuado que los ciudadanos hacen de dichos contenedores, sino la incorrecta disposición de espacios públicos para acumular las bolsas de basura de las casas.

Esta circunstancia provee de un contexto analizable desde las matemáticas, y fue aprovechada por los estudiantes del grado séptimo del CLC para acercarse al problema de las basuras y a la respuesta para la pregunta que dio origen al escenario: ¿Qué ocurre con las basuras que salen de nuestras casas? Este acercamiento incluyó una revisión documental de lo presentado por la UAESP respecto al esquema de recolección de basuras en la localidad de Bosa, del cual está encargada la firma LIME S.A ESP, particularmente lo relacionado con la normatividad aplicada a los esquemas de recolección, barrido y limpieza. El estudio se desarrolló durante los meses de febrero y marzo, por medio de la discusión en pequeños grupos de estudiantes acerca de lo presentado en el documento Comportamiento de la recolección de basuras en Bogotá durante el período de transición (primer semestre de 2018), presentado el mismo año por la Veeduría Distrital.

La discusión generó algunas interpretaciones frente a la cantidad de basura producida diariamente, apoyadas en la elaboración de gráficos y tablas de datos (Figura 1 y Figura 2) para representar las toneladas de residuos dispuestos en el Relleno Sanitario de Doña Juana durante enero 2017 y enero de 2018; así mismo, el trabajo permitió establecer comparaciones entre las diferentes cantidades de basura generadas, y reflexiones sobre la forma en ello afecta a nuestra comunidad. 
-educación

Figura 1. Registro en tabla de toneladas de basura producidas en Bogotá en 2018, realizada por estudiantes

\begin{tabular}{|c|c|c|c|c|c|}
\hline \multicolumn{3}{|c|}{ Enero 2017} & \multicolumn{3}{|c|}{ Enero 2018} \\
\hline Dia & Fecha & Toneladas & Dia & Fecha & Toneladas \\
\hline Domingo & 1 & 1.750 & Domingo & 1 & 4.400 \\
\hline Lunes & 2 & 6.250 & Lunes & 2 & 6.300 \\
\hline Martes & 3 & 6.700 & Martes & 3 & 7.50 \\
\hline Miércoles & 4 & 7.350 & Miércoles & 4 & 6.500 \\
\hline Jueves & 5 & 5.850 & Jueves & 5 & 6.050 \\
\hline Viernes & 6 & 6.200 & Viernes & 6 & 6800 \\
\hline Sábado & 7 & 5.800 & Sábado & 7 & 2.250 \\
\hline Domingo & 8 & 1.900 & Domingo & 8 & 5.400 \\
\hline Lunes & 9 & 5.600 & Lunes & 9 & 7.400 \\
\hline
\end{tabular}

Al tiempo, fue posible construir gráficos de compaen relación con fechas de consumo, tales como fines ración de datos y de análisis de tendencias, además de año y fiestas de reyes.

de interpretaciones sobre la producción de basuras

Figura 2. Análisis elaborado por estudiantes respecto al manejo de basuras

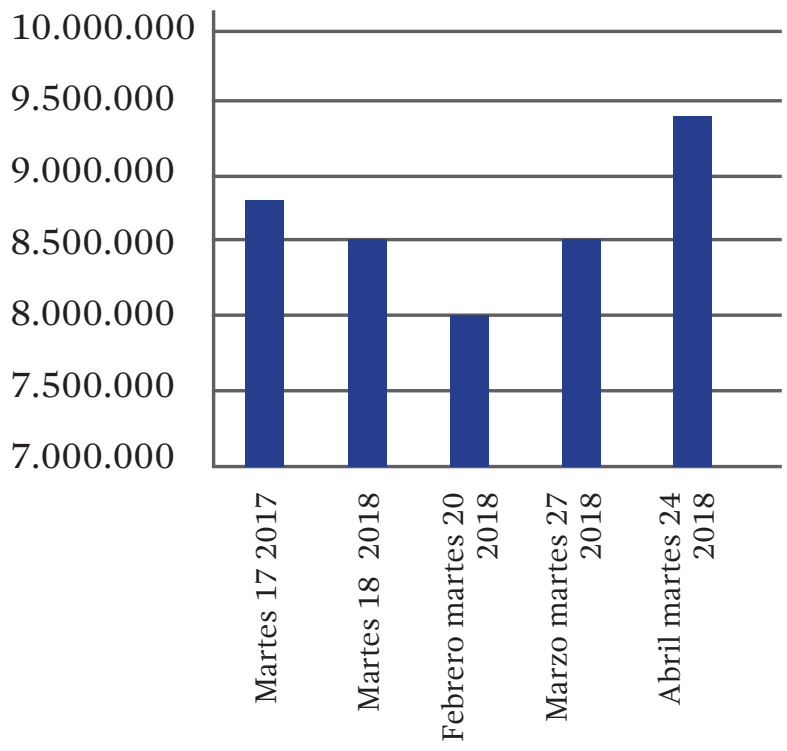

Vemos que en el 2018, en los meses de febrero, marzo $\mathrm{y}$ abril se han sacado cantidades de basura dispuestas por el Botadero Doña Juana.

En febrero las cantidades más altas de residuos son de 8.67, el día martes 20; en marzo sus cantidades más altas son de 8.496.42, del día martes 27; en abril la cantidad de basuras aumenta sus cifras, son de 9.410.10, del día 24 .

Nos podemos dar cuenta que los días martes las personas sacan más basura, y que del año 2017 al 2018 han disminuido y aumentado la cantidad de basura en Colombia. 


\section{Escena 2. Ordenar las basuras}

Sin duda, el primer referente para hablar del problema en cuestión es cada casa, por el cual, luego de lograr conciencia frente a la dimensión de basura generada en Bogotá, se precedió a indagar la cantidad de bolsas de basura producidas semanalmente en las casas que habitamos. Un ejercicio de registro simple dadoen el salón de clases luego de la revisión documental; permitió reconocer que, en promedio, semanalmente se producen tres bolsas de basura; también hizo posible destacar las prácticas enmarcadas en la disposición de los desechos, que fueron organizadas de la siguiente forma:

a. Separación de residuos. Al respecto se identificó que muy pocas casas realizan separación. Por el contrario, se saca una sola bolsa de basura con todos los residuos (incluidos los sanitarios) en las horas y días dispuestos para el paso del camión recolector.

b. Reciclaje. En algunos casos, los estudiantes afirman que sus padres trabajan como recicladores o sacan en una bolsa diferente aquello susceptible de ser reciclado.

c. Sacar la basura a una esquina. El diálogo con los estudiantes permitió concluir que en sus casas se saca la basura en horas de la mañana, dejándola en una esquina tácitamente dispuesta por la comunidad para dejar sus bolsas. Muchos manifestaron que esta situación genera distintos problemas, pues se dan molestias asociadas, como la presencia de roedores, malos olores y personas dedicadas al reciclaje que rompen las bolsas buscando materiales reutilizables.
Con lo anterior, apoyados en videos disponibles en la web, el grupo se dio a la tarea de comprender las formas como se propone hacer la separación de residuos en casa, y de formular hipótesis sobre lo que ocurriría si los hogares de la localidad hicieran un manejo adecuado de los residuos.

\section{Escena 3. Ideas para cambiar el mundo}

Debido a los procesos de cuarentena asumidos a nivel nacional, fue necesario continuar con el trabajo por medio de la plataforma Edmodo y de un grupo de WhatsApp, donde se compartía la información sobre las asignaciones de la clase; también se creó una página en Facebook para publicar las fotografías tomadas por los estudiantes o aportadas por otras personas de la comunidad educativa, para luego reflexionar sobre el problema de las basuras en la comunidad.

Precisamente, esas posibilidades favorecieron el desarrollo de un escenario compuesto por dos elementos, a saber: 1. Evidenciar el problema mediante fotografías; y 2. Establecer reflexiones frente al manejo de las basuras en nuestros barrios. Para el primer elemento, es posible destacar que en la página de Facebook, denominada "Entropía, escuela de matemáticas", reposan cerca de 72 fotografías tomadas por los estudiantes, padres de familia y profesor, evidenciando el mal manejo de basuras y la disposición inadecuada de espacios públicos para acumular bolsas de residuos provenientes de las casas.

Igualmente, se destacó una relación desfavorable de las personas con el ambiente, pues el registro fotográfico también prueba que en algunas zonas de la localidad, cercanas a las riveras del Río Tunjuelito y en terrenos baldíos o de propiedad de la Empresa de 
Acueducto y Alcantarillado de Bogotá (EAAB), fueron usadas como botaderos de materiales de construcción, muebles y derivados del petróleo, entre otros. Junto a ello, se pudo constatar que el reciclaje es una de las actividades económicas realizadas por miembros de la comunidad, pero, debido a la no separación de los residuos desde los hogares, algunos recicladores de oficio rompen las bolsas, dispersando los residuos y propagando olores y el derrame de líquidos lixiviados, derivados de la descomposición de basuras orgánicas.

Figura 3. Fotografía y reflexión expuesta en el proyecto "Entropía. Escuela de matemáticas"

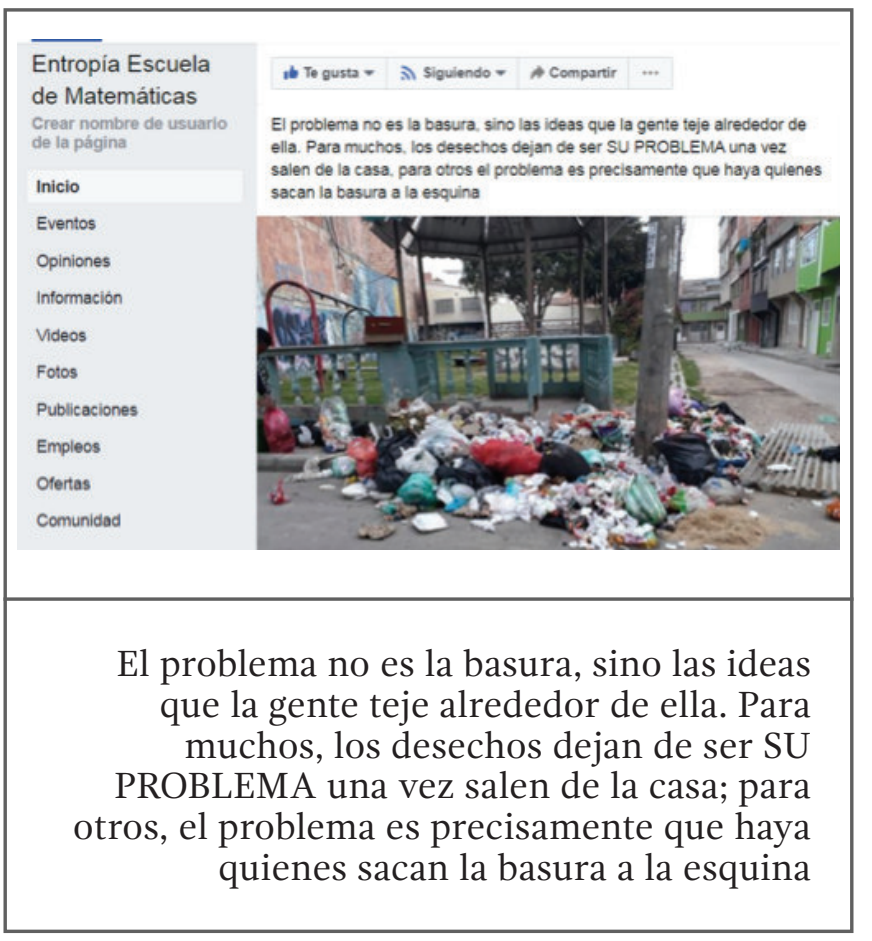

En cuanto al segundo elemento de reflexión, se destaca el hecho de que la publicación de fotografías en la página de Facebook permitió expresarse frente a la situación mediante comentarios en el pie de foto, estableciéndose diálogos referentes a las imágenes; ello propició lo que, en términos de Skovsmose (1999), se conoce como saber reflexivo, relacionado con la toma de una posición justificada en una discusión sobre asuntos tecnológicos. Es decir, al abordar el problema de las basuras, los estudiantes empiezan a reconocer la presencia de distintas variables que les impiden dar una solución simple, mientras, al tiempo, pueden emprender acciones para minimizar algunos impactos de esta mala práctica.

Para el ejercicio reflexivo, es importante resaltar que, dada su intención de referirse a las basuras, los estudiantes comenzaron a evaluar posibilidades enmarcadas en la modificación de prácticas, considerando la idea de establecer mecanismos de separación de basuras y un modelo que permitiera cuantificar la cantidad de desechos producidos por persona en casa, lo cual desató una multiplicidad de posiciones de acuerdo con las comprensiones de cada quien frente a la situación; por un lado, surgieron propuestas centradas en la necesidad de establecer una unidad de medida aplicable a todas las canecas dispuestas en las casas; una opción que encierra la dificultad de controlar la producción de basuras, dadas las dinámicas laborales y escolares de los miembros de cada familia; sin contar con que varía la cantidad de personas por hogar, y el trabajo está mediado por factores como las edades, la presencia de bebés en casa y el tipo de alimentos o bebidas consumidos. 
Por otra parte, algunas propuestas se dirigieron a establecer el tipo de residuos y la frecuencia con que se producen basuras, sin importar la unidad de medida. Lo cual implica dificultades frente a los instrumentos y prácticas de medición, la variedad de residuos y el desconocimiento sobre la forma como debe darse una separación adecuada; dichos planteamientos también expusieron la necesidad de emplear la información presentada en los recibos de servicios públicos respecto al cobro de recolección de basuras.

\section{Acciones propuestas}

Abordar una situación contextual como la producción de basuras, vista desde aspectos ambientales y matemáticos, configura discursos en torno a su posible solución, o aproximaciones a respuestas probables. En lo que sigue, se expondrán diferentes acciones desarrolladas por los estudiantes para responder a cuestionamientos surgidos durante la clase, aclarando que hay un conjunto de trabajos desarrollados por cada estudiante en su propia casa, y otros de orden colectivo, enmarcados en la discusión de lo desarrollado.

\section{Acción 1. Transformar el discurso de nuestras familias}

Para implementar esta acción, se comenzó por dialogar acerca de la necesidad de fomentar un manejo adecuado de los residuos en los propios hogares; ello implicó trasladar los aprendizajes logrados hasta concretarlos en acciones pedagógicas que permitieran comunicar a las familias algunas propuestas para realizar una adecuada separación de residuos. Así, se desarrolló un ejercicio de clasificación y separación de basuras en cada casa, liderado por los estudiantes y dirigido a sus familiares, para explicar el impacto de la separación en el hogar y el modo adecuado para realizarla; también se recolectó información sobre las dificultades presentes para implementar la separación de residuos en la casa.

\section{Acción 2. Clasificar las basuras, separarlas}

Una vez realizada la socialización con familiares de los estudiantes, se procedió a establecer la clasificación y separación de las basuras como una práctica cotidiana en cada una de las casas. Para ello, los estudiantes, junto con sus familias, dispusieron de un lugar en sus hogares para situar canecas de basura que funcionaran como espacio para la separación; de modo paralelo, empezaron a transformarse las prácticas de disposición de las bolsas de basura en los contenedores y lugares usados para tal fin por las comunidades.

Figura 4. Separación de residuos

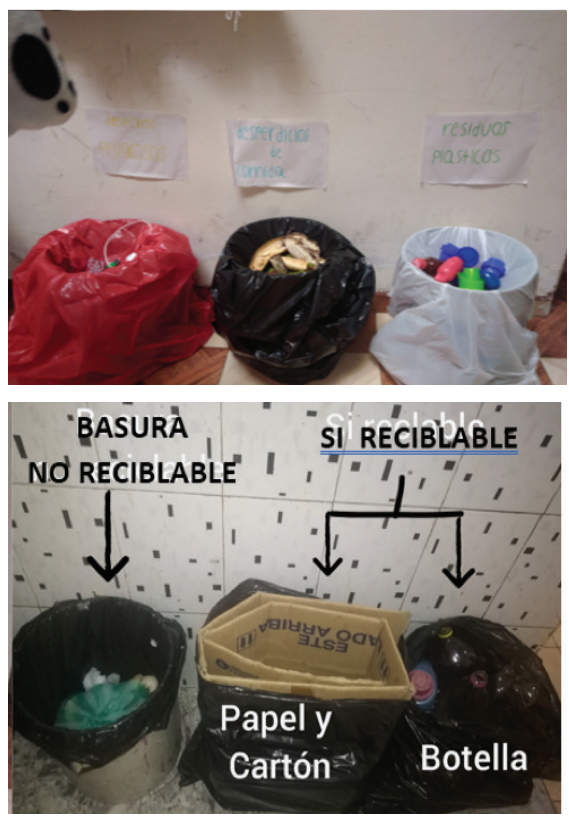


-educación

Es importante destacar que el ejercicio reflexivo de los estudiantes también tuvo como resultado la expresión de consideraciones alrededor de las dificultades y aciertos surgidos al momento de separar los residuos; a continuación, se presentan algunos de los relatos reunidos mediante un formulario en Google Forms:

Relato1: Solo tuvimos un problema. Faltaba que no teníamos basureros y tuvimos que dejar las bolsas en el suelo; considero que es necesaria la clasificación de las basuras, porque lo pueden volver a transformar en otra cosa, en el reciclable y en los orgánicos para la naturaleza.

Relato2: Las dificultades que tuvimos fueron que uno, cuando iba a echar la basura, los confundía y echaba la basura en la caneca que no era, y tocaba sacarla y echarla donde era o preguntar dónde se echa antes de echar la basura donde no era.

Relato 3: No se tuvieron inconvenientes en la familia, ya es algo que se vuelve mecánico, dónde se debe colocar cada cosa. Es importante reciclar, ya que con esto ayudamos al medio ambiente, ayuda al cambio climático, ahorramos energía, hay menos contaminación del aire. Al reciclar todos los plásticos y el papel no habrá necesidad de hacer tanto uso de materias primas para fabricar; de esta manera, ahorramos recursos naturales y preservamos nuestros bosques. Además, se reutilizan cosas que pensamos no servirán.

Como se puede observar, las narrativas presentadas permiten reconocer elementos que dan cuenta de la apropiación de conocimientos frente a la separación y clasificación de residuos; también se hacen evidentes las reflexiones en torno a la incidencia de las acciones ejecutadas y sus posibles impactos, además de reconocer una variación en las comprensiones del ambiente y un compromiso con la proposición de estrategias para mejorar el propio entorno.

\section{A modo de cierre}

El trabajo con los estudiantes, junto a lo expuesto en el presente documento, facilita una reflexión alrededor del papel de las matemáticas en el reconocimiento de la necesidad de disminuir la cantidad de residuos producidos en casa, particularmente para el grado séptimo del CLC. En cierto modo, los elementos de discusión enmarcados en el estudio estadístico de la variable "cantidad de basuras", constituyen un elemento que contribuye al debate en torno al ambiente y el impacto de las acciones humanas en su preservación.

La revisión de datos y su discusión permitieron reflexionar sobre la necesidad de hacer una adecuada separación de residuos en casa, partiendo de la idea de la reutilización de materiales reciclables y de la posibilidad de disminuir los desechos. Al tiempo, se desarrollaron reflexiones sobre las posibilidades, dificultades y utilidades de implementar estrategias de separación.

En tal sentido, es importante anotar que, luego del trabajo desarrollado, la transformación de las narrativas estudiantiles da cuenta de que los elementos utilizados, desde las matemáticas, potencian la reflexión y dinamizan la apropiación de la idea de lo ambiental en tanto proceso de construcción y relación permanente con el medio, mientras favorecen la aparición de actitudes, compromisos y competencias propias de la educación ambiental.

Por otro lado, es importante considerar que los espacios de discusión virtuales y presenciales, trabajados durante el proyecto, permiten visualizar el papel de las experiencias de los individuos en su 
comportamiento social; así, cuando un sujeto actúa sobre una situación no solo la ve como un marco de acción, la interpreta desde miradas externas que relativizan lo que ha considerado como cierto. Precisamente, en el escenario planteado, los estudiantes confrontan sus ideas sobre el manejo de basuras y llegan a consensos luego de escuchar o leer las consideraciones de otros.

El acuerdo sobre la forma de analizar la información, cómo desarrollar los registros, qué preguntar y privilegiar para el marco propuesto del escenario "basuras", revela el papel de la experiencia de los estudiantes frente a la forma de dar cuenta de los datos, mientras, al tiempo, expone que el abordaje de situaciones contextuales, desde la asignatura escolar de matemáticas, descentra las prácticas tradicionales, pues las respuestas relativizan los modos de resolver y las formas de comunicar los datos.

Ante la posibilidad de realizar acciones que permitan frenar el deterioro medioambiental, el camino queda abierto, al igual que la discusión sobre la forma de abordar el uso razonado de recursos y el manejo adecuado de los desechos; para responder a una problemática que, a pesar de enmarcarse en la escuela, tiene su reflejo en la calle. Esto ratifica la idea de fomentar la educación ambiental desde un ámbito interdisciplinar que contribuya a la toma de conciencia sobre los impactos de las interacciones del ser humano con el ambiente del cual hace parte.e. 


\section{Referencias}

Duarte, J. (2014). Estrategias alternativas para la resignificación del concepto ambiente en el CED La Concepción. Reconocimiento social a maestras y maestros. Bogotá: IDEP-Secretaría de Educación del Distrito.

Duarte, J., Urazán, R., Quiroga, M., Revelo, F., Guevara, A., y Gómez, N. (2019). Planeación de área de ciencias naturales desde preguntas orientadoras contextuales en el IED La Concepción. Revista Biografía, pp. 1003-1016.

García, O. (2017). Formación de jóvenes como líderes ambientales: el caso del CED La Concepción de Bogotá. Revista Bio-grafía, pp. 610-618. DOI: https:// doi.org/10.17227/bio-grafia.extra2017-7156

Morales, R. (2017). La consideración por el Otro en la clase de matemáticas. Un estudio desde la perspectiva de la educación matemática crítica. Tesis de Maestría no publicada, Facultad de Educación, Énfasis en Educación Matemática, Universidad Distrital Francisco José de Caldas.

Sauvé, L. (2010). Investigación didáctica. Educación científica y educación ambiental: Un cruce fecundo. Enseñanza de las ciencias. Revista de investigación y experiencias didácticas, Vol. 28(1).

Sauvé, L., y Orellana, I. (2001). La formación continua de profesores en educación ambiental: la propuesta de EDAMAZ. En Michéle, S. La contribución de la educación ambiental a la esperanza de Pandora (pp. 273-288). Sâo Carlos: Rima.
Skovsmose, O. (1999). Hacia una filosofía de la educación matemática crítica. Bogotá: Una Empresa Docente.

Skovsmose, O. (2000). Escenarios de investigación. Revista EMA, No. 6(1), pp. 3-20.

Skovsmose, O., y Valero, P. (2012). Rompimiento de la neutralidad política: el compromiso crítico de la educación matemática con la democracia. Educación matemática crítica: una visión sociopolítica del aprendizaje (pp. 1-24). Bogotá: Ediciones Uniandes.

Veeduría Distrital de Bogotá. (2018). Comportamiento de la recolección de basuras en Bogotá durante el período de transición (primer semestre de 2018). Obtenido desde http://veeduriadistrital.gov.co/sites/default /files/files/Publicaciones\%202018/Comportamie nto\%20de\%20la\%20recolecci\%C3\%B3n\%20de\%20 basuras\%20 en \%20Bogota\%20VF\%20( $07 \% 20$ nov\%2018).pdf 